\title{
Unruh effect in heavy ion collisions
}

\author{
Maksym Teslyk $^{1, a}$, Evgeny Zabrodin ${ }^{2,3,4}$, and Larisa Bravina ${ }^{2}$ \\ ${ }^{1}$ Faculty of Physics, Taras Shevchenko National University of Kyiv, 03022, Kyiv, Ukraine \\ ${ }^{2}$ Department of Physics, University of Oslo, PB 1048 Blindern, Oslo, Norway \\ ${ }^{3}$ Skobeltsyn Institute of Nuclear Physics, Moscow State University, RU-119991 Moscow, Russia \\ ${ }^{4}$ National Research Nuclear University "MEPhl" (Moscow Engineering Physics Institute), Kashirskoe high- \\ way 31, Moscow, RU-115409, Russia
}

\begin{abstract}
We apply the idea of the Unruh effect to the description of particle production in relativistic heavy ion collisions. Because the full information about all particles is needed, we employ the spectra of hadrons generated by the UrQMD model for $p p$ and $\mathrm{Au}+\mathrm{Au}$ collisions at various energies. Particles are considered as generated straight at the Unruh horizon thus allowing estimation of the Unruh temperature of the source. The analysis is provided for different types of mesons and their charges.
\end{abstract}

\section{Introduction}

In high energy collisions system quickly forgets its initial conditions after the relaxation to the completely thermal state, which is well described by hydrodynamic models in later times. The only quantities to be saved are the total ones, such as energy, angular momentum, charge etc. In order to make the models work, any other information about the constituents should be lost by the time of order of $1 \mathrm{fm} / c$ right after the collision. This is too small to be described within any kinetics available. Such a relaxation is known as fast thermalization, and even to date there is no any complete theoretical explanation of the process.

Unruh effect [1] is one of the candidates to explain the fast thermalization. It states that the noninertial observer experiences thermal radiation with temperature $T$, known as Unruh temperature,

$$
T=\frac{\alpha}{2 \pi}
$$

whereas the inertial one detects vacuum; here $\alpha$ is the acceleration of the observer.

The idea to apply Unruh effect for thermalization was proposed in [2]. Any creation of particle requires some amount of energy and therefore should lead to deceleration of the initial particles during the scattering process. The decelerating particle may be considered as the observer in the noninertial frame of reference (NFR) experiencing the Unruh radiation. The created particles are emitted in a completely thermal state from the horizon. Within this formalism, thermalization is a simple consequence of the influence of the horizon as a thermal bath.

\footnotetext{
a e-mail: machur@ukr.net
} 
The approach has been developed further in $[3,4]$, where the deceleration and particle creation were considered via the string tension and the string breaking mechanism. Comparison of the approach to the experimental data can be found in [5].

Further research on the topic is presented in [6], where author analyzes $\mathrm{P}$ and $\mathrm{CP}$ violation via the Unruh mechanism. In [7] the discrepancy between this approach and the RHIC data on photon radiation was revealed. In [8] the authors have tried to identify the parameters of the black hole, which reproduces yields and energy spectra of particles emitted in high energy scattering processes at Hagedorn temperature.

Thus we conclude that the Unruh effect might be a promising approach to solve the early thermalization problem. However there is a need in further investigations. In the present paper we propose the method of verification of the Unruh effect by means of the multiplicity and energy distributions. Using the UrQMD $[9,10]$ calculations as a data source, we investigate the distributions for different types of mesons and their electrical charges, namely, $\pi, \eta, K$ and all of them in order to calculate the temperature of the process. In contrast to [3-5] we do not analyze the string breaking mechanism directly. Instead, we suggest appearance of the Unruh effect because of the momentum exchange between the colliding particles, without the direct analysis of the scattering process. The horizon, once appeared, is considered as an entropy source for the system and as an origin of the thermalization. The source generates distributions of the quantities which are being measured and hence allow to estimate its temperature.

\section{Unruh effect}

In what follows we consider particles with spin 0 ; the other spin cases need a bit more complicated analysis which is beyond of the scope of this manuscript.

Let us consider some pure vacuum state $|\phi\rangle$ in the inertial frame of reference (IFR).

$$
a|\phi\rangle=0,
$$

where $a$ is the annihilation operator in the IFR. The situation looks different in the non-inertial frame of reference determined by acceleration $\alpha$. There is some spacetime region in the IFR's lightcone, the signals from which cannot reach the observer from the NFR at any times. This may be interpreted as appearance of some kind of the event horizon: the NFR observer cannot obtain the information about the internal of the region till the acceleration lasts. As a result, the state (2) in NFR will take the form (see $[11,12]$ for details)

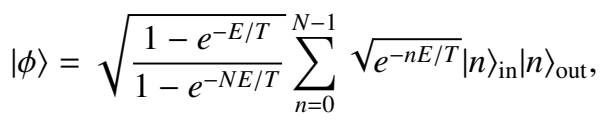

where $E$ is the energy of the particles in the NFR, $T$ is the Unruh temperature determined by (1), and subscripts in(out) denote modes in(out)side with respect to the horizon; $N-1$ determines maximum number of particles which may be generated. Since the inside mode is inaccessible, one should trace out over its degrees of freedom, and thus result in the state

$$
\rho_{\text {out }}=\operatorname{Tr}_{\text {in }}|\phi\rangle\left\langle\phi\left|=\frac{1-e^{-E / T}}{1-e^{-N E / T}} \sum_{n=0}^{N-1} e^{-n E / T}\right| n\right\rangle_{\text {out }}\langle n|
$$

that describes the radiation going out from the horizon and being detected with the NFR observer (Unruh effect). Expression (4) represents multiplicity distribution of spinless particles at given energy $E$ and temperature $T$ with von Neumann entropy

$$
H\left(\rho_{\text {out }}\right)=\ln \frac{1-e^{-N E / T}}{1-e^{-E / T}}+\frac{E / T}{e^{E / T}-1}-\frac{N E / T}{e^{N E / T}-1} .
$$




\section{Model}

Firstly, we recall some items from the probability theory. Let us consider some distribution $\{X, Y\}$ of quantities $x, y$ with the probability $p(x, y), \sum_{x, y} p(x, y)=1$. Shannon entropy of the distribution is then

$$
H(X, Y)=-\sum_{x, y} p(x, y) \ln p(x, y)
$$

Conditional probability $p(x \mid y)$ may be written as

$$
p(x \mid y)=\frac{p(x, y)}{p(y)}, \quad p(y)=\sum_{x} p(x, y) .
$$

It defines probability of $x$ being given $y$.

Substituting (7) to (6) and using that $p(x, y)=p(y, x)$ one obtains

$$
H(X, Y)=H(Y)+\langle H(X \mid y)\rangle_{y}=H(X)+\langle H(Y \mid x)\rangle_{x},
$$

where averaging means $\langle\bullet\rangle_{y}=\sum_{y} p(y) \bullet$ and

$$
H(X \mid y)=-\sum_{x} p(x \mid y) \ln p(x \mid y)
$$

is the Shannon entropy for conditional distribution $\{p(x \mid y)\}$.

From (4) it follows that Unruh effect may be interpreted as a conditional distribution process, because the eigenvalues of $\rho_{\text {out }}$ determine conditional multiplicity distribution $\{p(n \mid E / T)\}$. Quantities $n$ and $E$ can be directly extracted from the measurements, and $T$ is an unknown to be found. In order to estimate temperature one should solve equation (8), i.e.

$$
\langle H(n \mid E / T)\rangle_{E / T}=H(n)+\langle H(E / T \mid n)\rangle_{n}-H(E / T),
$$

where

$$
H(n \mid E / T)=H\left(\rho_{\text {out }}\right),
$$

see (5). In addition one should fix the parameter $N$ to complete the equation.

The whole algorithm is as follows:

1. Extract multiplicity $n$ and energy $E$ spectrum data for different particle types of spinless bosons from collision data to construct the all necessary distributions.

2. Set parameter $N$, see (4), taking into account the conservation laws. Usually it is assumed that $N=\infty$. However, this is not the correct choice because of violation of conservation laws (energy, electrical, baryonic and other charges) during the collision.

3. Substitute parameters obtained from the distributions and conservation laws to (11). One may prefer to solve (11) directly, i.e. to make the simple fitting. However, such an approach seems to generate additional error: it requires to fit distribution for every energy range. As a result, one will obtain a set of temperatures which should be averaged to exclude energy dependence, and thus will lead to additional error.

4. Construct and solve equation (10) to extract the temperature. Taking into account the all possible energy values seems to be difficult due to the limitations of the computer time, and therefore there is a need in splitting the whole energy range to the bins and using average energy values in every bin. However, equation (10) is valid and exact for any energy distribution, and therefore solving (10) instead of fitting (11) does not generate additional error. 


\section{Results}

In this section we present the results obtained according to the algorithm from Section 3. In our analysis we used UrQMD calculations instead of experimental data in order to verify the whole principle and the possibility to keep tracking the model in dynamics. The calculations were made for $\mathrm{Au}+\mathrm{Au}$ collisions for the set of energies $\sqrt{s}=\{50,100,150,200\} \mathrm{AGeV}$. We analyzed the cases of $\pi, \eta, K$ mesons, respectively, and of combined spectrum of all these mesons. In order to investigate the influence of electrical charge on the effect we calculated separately neutral (indexed by ${ }^{0}$ ), charged (indexed by ${ }^{\text {ch }}$ ) and all (neutral + charged) particles. The parameter $N$ is assumed to be $N=\max (\{n\})+1$, i.e. the maximal multiplicity of the whole data set for the given $\sqrt{s}$ plus one.

To explore the properties of entropy $H\left(\rho_{\text {out }}\right)$ we present graphically some of the constructed functions. The entropy was averaged over the energy distribution, what is denoted by $\langle\bullet\rangle_{E}$. We do not present all the cases calculated since they are expected to demonstrate the similar behaviour.

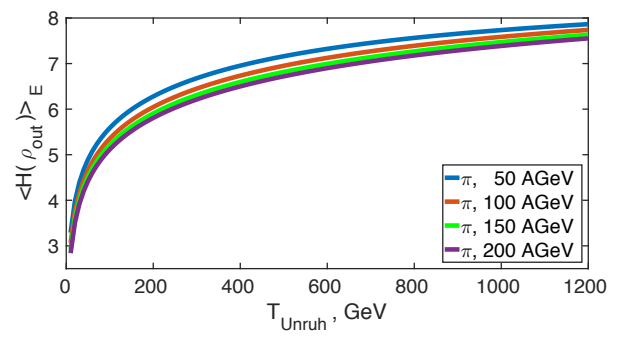

(a) $\pi$

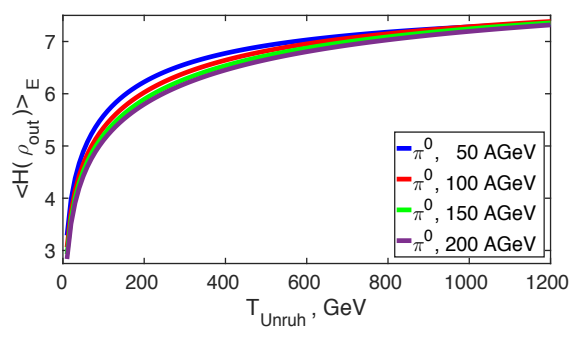

(b) $\pi^{0}$

Figure 1: Dependence of $\left\langle H\left(\rho_{\text {out }}\right)\right\rangle_{E}$ on $T$ after freeze-out in UrQMD calculations of Au+Au collisions at different $\sqrt{s}$

In Fig. 1 we depict dependence of entropies $\left\langle H\left(\rho_{\text {out }}\right)\right\rangle_{E}$ on temperature of the Unruh source (denoted by Unruh) $T$ at different $\sqrt{s}$ for $\pi$, see (a), and for $\pi^{0}$, see (b). As expected, the entropy is growing with the increasing temperature. However, it grows very fast at temperatures below $150 \mathrm{GeV}$. At higher temperatures the slopes of the distributions are not so steep. It is worth mentioning the inverse energy hierarchy within the presented temperature range $T \leq 1.2 \mathrm{TeV}$. Namely, the larger the collision energy, the smaller the entropy. For neutral pions all distributions seem to converge at $T>800 \mathrm{GeV}$.

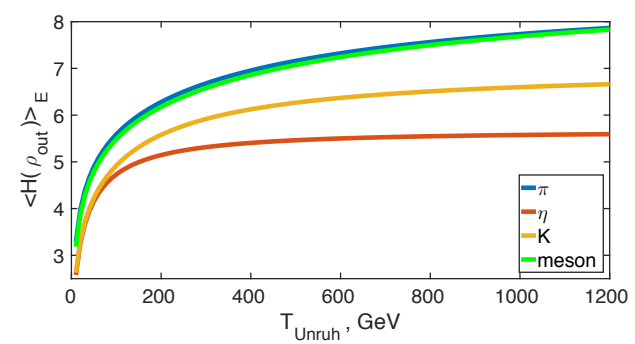

(a) $\pi, \eta, K$, mesons

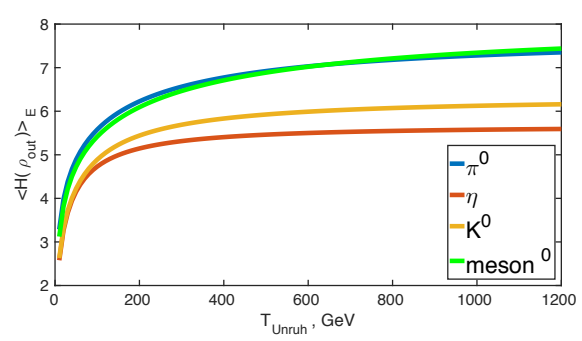

(b) $\pi^{0}, \eta, K^{0}$, mesons ${ }^{0}$

Figure 2: Dependence of $\left\langle H\left(\rho_{\text {out }}\right)\right\rangle_{E}$ on $T$ for different meson types after freeze-out in UrQMD calculations of $\mathrm{Au}+\mathrm{Au}$ collisions at $\sqrt{s}=50 \mathrm{AGeV}$ 
In Fig. 2 we present the plots of $\left\langle H\left(\rho_{\text {out }}\right)\right\rangle_{E}$ depending on $T$ at collision energy $\sqrt{s}=50 \mathrm{AGeV}$ for different meson species. $\pi, \eta, K$ and their combined spectrum (denoted as 'meson') are depicted in Fig. 2(a), whereas $\pi^{0}, \eta, K^{0}$ and all of them (denoted as 'meson' ${ }^{0}$ ') are depicted in Fig. 2(b). Again, the entropy increases with rising temperature. Entropies of pions and of all mesons almost coincide irrespective on the electric charges, because the total yields of both charged and neutral mesons are heavily dominated by pions.

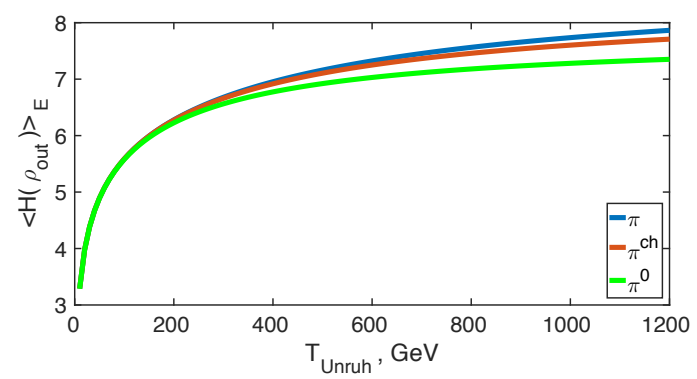

Figure 3: Dependence of $\left\langle H\left(\rho_{\text {out }}\right)\right\rangle_{E}$ on $T$ for $\pi, \pi^{\mathrm{ch}}, \pi^{0}$ after freeze-out in UrQMD calculations of $\mathrm{Au}+\mathrm{Au}$ collisions at $\sqrt{s}=50 \mathrm{AGeV}$

Comparing (a) and (b) cases for Fig. 1 or Fig. 2, one can notice the dependence of $\left\langle H\left(\rho_{\text {out }}\right)\right\rangle_{E}$ on the electrical charge, that can be seen clearly in Figure 3. Here we presented dependence of $\left\langle H\left(\rho_{\text {out }}\right)\right\rangle_{E}$ on $T$ at $\sqrt{s}=50 \mathrm{AGeV}$ for $\pi, \pi^{\mathrm{ch}}$ and $\pi^{0}$. All three spectra demonstrate similar behaviour, but grow with a bit different rates at $T_{\text {Unruh }}>200 \mathrm{GeV}$. $\left\langle H\left(\rho_{\text {out }}\right)\right\rangle_{E}$ for all pions shows the largest growth rate, while for the neutral pions the growth rate is the slowest one. This might be the consequence of the amount of particles taken into account for the analysis.

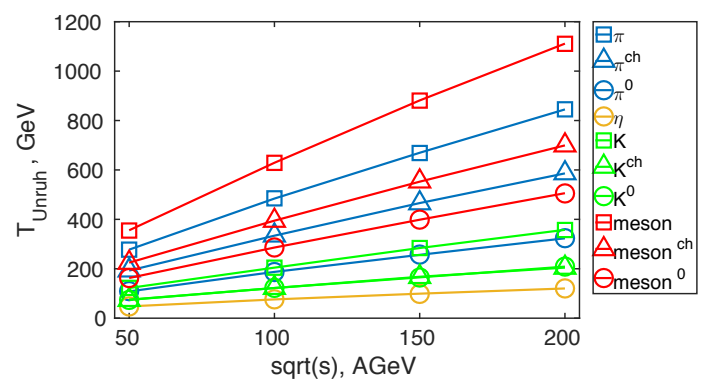

Figure 4: Dependence of $T$ on collision energy $\sqrt{s}$ and charge for $\pi, \eta, K$, mesons after freeze-out in UrQMD calculations of $\mathrm{Au}+\mathrm{Au}$ collisions. Lines are to guide the eye.

The final result is presented in Fig. 4. Here the temperature $T$, calculated as a solution of Eq. (10), is plotted versus collision energy $\sqrt{s}$ for different meson species. The statistical error bars are small and lay within the plotted symbols.

The temperature of the Unruh source has appeared to be in range $O(10) . . O\left(10^{3}\right) \mathrm{GeV}$. At the first glance, $T$ seems to increase with $\sqrt{s}$ linearly. But more accurate analysis shows that this is not the case. 
All particle spectra except those for $K^{\text {ch }}$ and $K^{0}$ demonstrate different slopes of the distributions. Neutral mesons possess the lowest slopes compared to charged or combined meson spectra. The latter show the strongest increase of $T_{\text {Unuh }}$ with the collision energy $\sqrt{s}$.

The case $N=\max (\{n\})+1$ seems to be not good enough, because the total energy used for particle production of some specific meson type may be larger than it is allowed by the energy conservation law. In order to improve the estimations further, one should use $N=\max \left(\left\{n_{E}\right\}\right)+1$, i.e. take the maximum multiplicity at given energy $E$ instead. However, the last case seems to provide no solution of equation (10) for any meson type from both $\mathrm{Au}+\mathrm{Au}$ and $p p$ collisions at $\sqrt{s}$ within the indicated energy range. The possible reasons and the ways to overcome the problem are discussed in the next Section.

\section{Conclusions}

We developed the algorithm to extract Unruh temperature from the heavy ion collision data. The method was verified using the UrQMD simulations for Au+Au and $p p$ collisions at different $\sqrt{s}$. The results allow for two different interpretations:

- at given multiplicity and energy distributions, Unruh source with temperature $T$ is equivalent to the corresponding collision experiment;

- particles are born in complete thermal equilibrium by the Unruh source at temperature $T$. In other words, the created particles should be described by the mixed density matrix state parameterized by temperature $T$.

Usually, the parameter $N$ is assumed to be infinite. However, this is not the case in any scattering process since it requires infinite energy to create an infinite number of particles and violates the conservation laws. For $\mathrm{Au}+\mathrm{Au}$ collisions the parameter $N$ was chosen to be $N=\max (\{n\})+1$. For this value the estimated temperature $T$ of the Unruh source is presented in Fig. 4. From the estimation it follows that $T \gg T_{\text {Hagedorn }}$ : it is of the order of $O\left(10^{1}\right) . . O\left(10^{3}\right) \mathrm{GeV}$ and increases with $\sqrt{s}$.

At the first glance, this seems to be a contradiction discarding off the model presented. However, a hot source may be a good candidate for the thermalization. Such a high temperature may be explained by an analogy with the Sun, where the initial energy generating process, i.e. fusion, is of the order of $10^{6} \mathrm{eV}$ range, while the outgoing radiation from the Sun is within the $1 \mathrm{eV}$ range.

One may argue that such a high temperature should be explained by the influence of the environment that leads to broadening (fuzzing) of the distributions and hence to increasing of the whole entropy. The assumption is supported by the fact that temperature is higher for the combined meson spectra than for any particular meson species, see Fig. 4. In order to verify it one should consider the two-particle collisions, such as $p p$ or $\mathrm{e}^{+}+\mathrm{e}^{-}$.

The case $N=\max (\{n\})+1$ violates the energy conservation law, since it is independent on the energy $E$ of the created particles. The improved analysis with $N=\max \left(\left\{n_{E}\right\}\right)+1$ both for $\mathrm{Au}+\mathrm{Au}$ and for $p p$ collisions revealed that there are no solutions of (10) for this value of $N$. Substitution of the Planck temperature $T=T_{\text {Planck }}$ into (11) indicates that in (10) the left hand side does not exceed $40 \div 60 \%$ of the right hand side then.

Thus we conclude that the Unruh effect may not be responsible for the whole entropy generated in the collision. Even in the case of $p p$ collisions the average collision number is larger than one per event. This implies the presence of some kinetics that broadens the distributions and thus washes out the effect. In order to exclude the kinetics one should consider the time dependence of the Unruh effect during the collision, which is the next step in our research. 


\section{References}

[1] W.G. Unruh, Phys. Rev. D 14, 870 (1976)

[2] D. Kharzeev, K. Tuchin, Nucl. Phys. A 753, 316 (2005)

[3] H. Satz, Prog. Theor. Phys. Supplement 168, 338 (2007)

[4] P. Castorina, D. Kharzeev, H. Satz, Eur. Phys. J. C 52, 187 (2007)

[5] F. Becattini, P. Castorina, J. Manninen, H. Satz, Eur. Phys. J. C 56, 493 (2008)

[6] A.R. Zhitnitsky, Nucl. Phys. A 853, 153 (2011)

[7] T.S. Biro, M. Gyulassy, Z. Schram, Phys. Lett. B 708, 276 (2012)

[8] P. Castorina, D. Grumiller, A. Iorio, Phys. Rev. D 77, 124034 (2008)

[9] S. Bass, M. Belkacem, M. Bleicher, M. Brandstetter, L. Bravina, C. Ernst, L. Gerland, M. Hofmann, S. Hofmann, J. Konopka et al., Progr. Part. Nucl. Phys. 41, 255 (1998)

[10] M. Bleicher, E. Zabrodin, C. Spieles, S.A. Bass, C. Ernst, S. Soff, L. Bravina, M. Belkacem, H. Weber, H. Stocker et al., J. Phys. G 25, 1859 (1999)

[11] R. Banerjee, B.R. Majhi, Phys. Lett. B 675, 243 (2009)

[12] D. Roy, Phys. Lett. B 681, 185 (2009) 